were in compiling the references. The text itself is rather brief in relation to the apparatus of references and indexes; 248 pages of text, tables and figures could hardly be expected to describe the references cited, let alone discuss them to any degree of thoroughness. Many important studies are given only a few sentences, whereas others are mentioned in a phrase or are lumped with other related papers in an overall characterisation. There is not much critical discussion of experiments or conclusions offered by investigators, although the authors occasionally point out discrepancies among reports and suggest some factors that complicate research and interpretation.

The title and prologue of the book may set up too high expectations by indicating that ". . . a theory for the 'Behavioral Code' is provided . .." Probably the emphasis should be placed on the word "elements", and perhaps "ingredients" would have been a more appropriate term. Table 80 lists as "Elements of the Behavioral Code" items as diverse as environmental stimulation, initiation of nerve impulses and transmitter release, effects on rates of synthesis of cyclic nucleotides, changes in synaptic efficiency, and changes in learning, memory, and adaptive behaviour. Elsewhere in the book we are told that both shortterm and long-term memory are elements and that ". . . neurotransmitter suspects [that is, presumed neurotransmitters] are integral elements of the Behavioral Code."

Apparently, although no definition is given, the Code when completed will include all of the events and processes that must intervene between initial capture of sensory information and storage of memory. Even the retrieval of memories should be included, although this book reflects most of the work in the field by not even mentioning retrieval. DeFeudis and DeFeudis believe, as do many workers in this field, that storage of memory requires a complex series of processes rather than depending upon some one key process. Their book is a substantial contribution towards the eventual formulation of a comprehensive theory of the neural mechanisms of learning and memory.

Mark R. Rosenzweig

Mark R. Rosenzweig is Professor of Psychology at the University of California at Berkeley.

\section{Vertebrate visual system physiology}

Handbook of Sensory Physiology. Vol. VII/5: The Visual System in Vertebrates. Edited by F. Crescitelli. (Springer: Berlin, Heidelberg and New York, 1978.) DM 340; $\$ 149.60$

This volume contains contributions by Crescitelli, Dvorak, Eder, Granda, Hamasaki, Holmberg, Hughes, Locket, McFarland, Meyer, Muntz, Munz, Olson and Reyer. The subject matter is concerned with the morphology, optics, pigments, physiology and adaptive radiation of the eye in different vertebrate classes. The chapters are comfortably short; most are under one hundred pages in length.

This volume is both necessary and important as a supplement to the earlier volumes in the Handbook. It is a necessary text for those libraries which deal with visual science, whether in its medical, biological or behavioural aspects, and is important in providing a source-book which is reasonably comprehensive and broad in outlook. It is occasionally suggested that large texts are on the verge of extinction because of the rate of publication of new papers, and because they can be compendious rather than critical. The latter comment applies only in part to this volume, where a few sections might have been sign-posted better, and where the literature might have been treated more sceptically. Most research workers in vision could find flaws and omissions where their speciality has been touched on, yet would surely benefit from and be stimulated by the breadth and scope of this book.

Although it is probably unfair to pick out single contributions for comment, for all the chapters are of high quality, I was particularly fascinated by the treatment given by Hughes to comparative optics. This chapter shows how much more there is to retinal organisation in mammals than the cat, and is essentially a modern critical essay in the spirit of Gordon Walls.

I applaud this book, and hope that despite its price, it will be widely appreciated by students and research workers interested in vision.

A. L. Holden

A. L. Holden is at the Institute of Ophthalmology, University of London, $U K$

\section{Spectroscopic techniques}

The Spectrum in Chemistry. By J. E. Crooks. Pp. 313. (Academic: London, New York and San Francisco, 1978). Hardback $£ 12.60$; paperback $£ 6.50$.

This is a straightforward textbook on spectroscopic techniques which are used in modern chemistry. All regions of the electromagnetic spectrum are covered, and the book is stronger than most of its rivals in the areas of X-ray spectroscopy, of photoelectron spectroscopy and of dispersion and dichroism associated with optically active materials. Indeed, chapter 9, which introduces optical activity through helices rather than asymmetrically substituted carbon atoms, is especially clear and a sufficient reason to recommend the work to undergraduate science libraries. The whole is well balanced and should be sufficient in depth for most British undergraduate courses. Thus, selection rules are briefly covered where appropriate but transition moments are not introduced. It is stronger on the general ideas and on spectroscopy in its own right than it is on quantum theory or on applications to organic or inorganic chemistry.

The author uses SI units, which is an attraction, although one wishes he did not so often remind the reader by attaching the units to the symbol for the physical quantity. For example, following equation (1.3), into which numerical values can be inserted using any set of coherent units, he adds :" $k$ is Boltzmann's constant in $\mathbf{J} \mathbf{K}^{-1 "}$. $\mathrm{J} \mathrm{K}^{-1}$ may prove convenient units but they are not essential and $k$ stands for Boltzmann's constant in any units. The book has some 20 questions with answers. It also has its share of blemishes and errors, but these are mostly misprints or details which are wrong and do not detract from the qualitative message; they would be more important to the practitioner than an average student.

It is interesting that, as far as the reviewer can discover, Dr Crooks' personal research interests have been in rapid reactions and in solute transfer with emulsions, so that he is not an established expert in spectroscopy. There is much argument as to whether experts or others are to be preferred as undergraduate textbook writers: this book shows the demerit of the inexpert with its occasional errors, but also shows the merit of clarity and balance to be sought from 'an outsider'.

D. H. Whiffen

D. H. Whiffen is Professor of Physical Chemistry at the University of Newcastleupon-Tyne, UK. 